\title{
Unified Analysis of Equal-Gain Diversity on Rician and Nakagami Fading Channels*
}

\author{
A. Annamalai ${ }^{1}$, C. Tellambura ${ }^{2}$ and V. K. Bhargava ${ }^{1}$ \\ 1. Department of Electrical and Computer Engineering, University of Victoria, PO Box 3055 STN CSC, Victoria \\ BC.V8W 3P6, Canada, Tel:+1-250-721-6043, Fax: +1-250-721-6048, E-mail: bhargava@ece.uvic.ca \\ 2. School of Computer Science and Software Engineering, Monash University, Clayton, Victoria 3168, Australia \\ Tel: +1-613-9905-3296,Fax: +1-613-9905-3402, E-mail: chintha@dgs.monash.edu.au
}

\begin{abstract}
Exact analytical expression in a simple form for computing the average symbol error rate (SER) of an arbitrary two-dimension signaling format with equal-gain diversity (EGC) receiver is not available - in the literature despite its practical and theoretical importance. The principle difficulty is finding a closed-form expression for the probability density function (PDF) of a sum of $L$ (i.e., diversity order) random fading amplitudes. In this paper, we develop an alternative, direct technique to evaluate the exact performance of EGC diversity systems (expressed in terms of a single or double finite-range integrals) in Rayleigh, Rician and Nakagami fading channels. Our new approach relies on the use of Parseval's theorem to transform the error integral into the frequency domain. Since the Fourier transform of the PDF is the characteristic function (CHF), which is available in this case, our solution is general and exact. The CHF method also circumvents the need to perform an $L$-fold convolution integral which is usually encountered in the calculation of the PDF of the sum of the received signal amplitudes. Interestingly, we can also get some new closed-form solutions for binary CPSK and CFSK in Nakagami fading channel for all $L \leq 3$. Closed-form formulas for binary DPSK and NCFSK with EGC may also be obtained for $L<3$.
\end{abstract}

\section{INTRODUCTION}

In recent years, multi-level modulation schemes have received considerable attention for facilitating high-rate data transmission over wireless links due to their inherent spectral efficiency. However, some form of diversity reception is usually needed to mitigate the effects of deep fades experienced on wireless channels as well as to reduce the penalty in signal-to-noise (SNR) associated with a larger alphabet size (i.e., denser signaling constellations) and the cochannel interference. Besides, diversity techniques play an important role in minimizing the transmit power requirements, particularly in the reverse link, because of the limited battery capacity of handheld subscriber units.

The EGC is of considerable interest since it appears to offer comparable performance to the optimal maximal-ratio diversity combining (MRC) with much greater simplicity, making it hardware feasible and cost viable. Despite its practical interest, exact analysis for EGC diversity receiver for the

\footnotetext{
* This work was supported in part by a Strategic Project Grant from the Natural Sciences and Engineering Research Council (NSERC) of Canada and in part by Telus Mobility.
}

general case of two-dimensional M-ary signalling in a generalized fading channel is not available in the literature. The principle difficulty is determining a closed-form expression for the PDF of the sum of random fading amplitudes. In fact, computing the PDF of the sum of multiple Rayleigh variable has been dilemma for many years, dating back to Lord Rayleigh himself, but has never been solved in terms of tabulated functions for $L \geq 3$ [1]. There is also no closed-form solutions for the PDF of a sum of Nakagami or Rician random variables (RVs). A detailed discussion of these problems are highlighted in [2].

Previous related studies on the EGC diversity include the following: In [3], Altman and Sichak have found an exact solution for the dual diversity system operating in a Rayleigh fading environment. For higher order of diversity, Jakes [1] has made use of a small argument approximation for the PDF suggested by Schwartz et. al. [4]. The small argument approximation method becomes inaccurate when $L$ increases. A more refined analysis was presented recently by Beaulieu in [2], where he devised an approximate infinite series technique to compute the PDF for the sum of independent Rayleigh RVs. Applying this technique, [5] and [6] analyze the performance of EGC for coherent and differential binary signalling schemes in Nakagami and Rician fading channels. Subsequently, Zhang [7] presented some closed-form solutions for binary signalling schemes in a Rayleigh fading channels. In [8], Simon and Alouini presented yet another approximate solution for the BPSK case on Nakagami fading channel using Hermite integration. Differently in [9], we have developed a direct technique to evaluate the exact performance of MQAM with EGC. Our new approach relies on the use of Parseval's theorem to transform the error integral into the frequency domain. Since the Fourier transform (FT) of the PDF is the $\mathrm{CHF}$ and the FT of the conditional error probability can be expressed in a closed-form, our solution is general and exact. Moreover, the resulting single finite-range integral can be approximated very precisely using the Gauss-Chebychev quadrature (GCQ) formula. More recently, Dong et. al. [10] provides an accurate analysis for six 16-ary signal constellations with EGC by using the approximate infinite series technique initially developed in [2].

By contrast, in this paper we generalize our previous results [9] by deriving an exact analytical expression for the SER of a broad class of coherent, differentially coherent and noncoherent modulation formats with predetection EGC. Sub- 
sequently, we show that the generic expression can be further simplified for four special forms of the conditional error probability. The contributions of this paper include: (a) derivation of simple yet exact analytical expressions for the SER of binary and two-dimensional signal constellations in Rayleigh, Rician and Nakagami fading channels with EGC; (b) derivation of a new expression for the CHF of the fading amplitude in Rician fading; (c) derivation of a rapidly converging series to precisely estimate the finite-range integral(s); (d) derivation of a desirable exponential form for the Gaussian probability integral which is suitable to perform averaging over the fading amplitudes; and (e) derivation of some new closed form expressions for coherent, differentially coherent and noncoherent binary modulation formats in Nakagami fading environment with integer fading severity index.

\section{STATISTICAL CHARACTERIZATION OF THE EGC} COMBINER OUTPUT

In an EGC combiner, the output of different diversity branches are first co-phased, equally weighted, and then summed to give the resultant output. The instantaneous SNR at the output of the EGC combiner is $\gamma=x^{2}$ where $x$ is defined as

$$
x=\sqrt{\frac{E_{b}}{L N_{0}}} \sum_{l=1}^{L} \alpha_{l}
$$

where $\alpha_{l}$ is the fading amplitude which may be modelled as a Rayleigh, Rician or a Nakagami RV, and $L$ denotes the diversity order.

\section{A. Nakagami Fading}

The CHF of $x$ in a Nakagami fading has been derived in [9] and is given by

$$
\begin{aligned}
\phi_{x}(\omega)=\prod_{k=1}^{L}\left\{\frac{\Gamma\left(m_{k}+1 / 2\right)}{2^{-m_{k}} \sqrt{\pi}} \exp \left(\frac{-\omega^{2}}{8 \lambda_{k} L}\right) D_{-2 m_{k}}\left(\frac{-j \omega}{\sqrt{2 L \lambda_{k}}}\right)\right\} \\
=\prod_{k=1}^{L}\left[\Phi\left(m_{k}, \frac{1}{2} ; \frac{-\omega^{2}}{4 L \lambda_{k}}\right)\right. \\
\left.+j \omega \frac{\Gamma\left(m_{k}+1 / 2\right)}{\Gamma\left(m_{k}\right) \sqrt{L \lambda_{k}}} \Phi\left(m_{k}+\frac{1}{2}, \frac{3}{2} ; \frac{-\omega^{2}}{4 L \lambda_{k}}\right)\right]
\end{aligned}
$$

where $m_{k}$ denotes the fading figure of the $k$-th diversity branch, $D_{-r}($.$) is the parabolic cylinder function of order v$, $\lambda_{k}=m_{k} / \bar{\gamma}_{k}$ where $\bar{\gamma}_{k}=\frac{E_{b}}{N_{0}} E\left\{\alpha_{k}^{2}\right\}=\frac{\Omega_{k} E_{b}}{N_{0}}$ corresponds to the average received SNR of the $k$-th branch, and $\Phi(a, b ; c)$ is the confluent hypergeometric function of the first kind. The confluent hypergeometric function can be computed efficiently using a convergent series for small arguments and via a divergent expansion for large arguments [9].

\section{B. Rician Fading}

The derivation of CHF of $x$ in Rician fading channel is slightly more involved compared to the Nakagami fading case because the PDF of the Rician RV contains an explicit term of a modified Bessel function of the first kind. Closed-form expression for the FT of the PDF is not known. Hence in [6], Abu-Dayya et. al. obtained an expression for $\mathrm{CHF}$ by replacing the Bessel function with its infinite series representation,

$$
\begin{aligned}
& \phi_{x}(\omega)=\prod_{k=1}^{L}\left[\operatorname { e x p } ( - K _ { k } ) \left[\sum_{i=0}^{\infty} \frac{K_{k}^{i}}{i !} \Phi\left(i+1, \frac{1}{2} ; \frac{-\bar{\gamma}_{k} \omega^{2}}{4 L\left(1+K_{k}\right)}\right)\right.\right. \\
& \left.\left.+j \omega \sqrt{2} \xi \sum_{i=0}^{\infty} \frac{\Gamma(i+3 / 2) K_{k}^{i}}{(i !)^{2}} \Phi\left(i+\frac{3}{2}, \frac{3}{2} ; \frac{-\bar{\gamma}_{k} \omega^{2}}{4 L\left(1+K_{k}\right)}\right)\right]\right]
\end{aligned}
$$

where $\xi=1 / \sqrt{2 L\left(1+K_{k}\right) / \bar{\gamma}_{k}}$.

By contrast, here we exploit the exponential integral representation for $I_{0}($.$) and therefore the individual CHFs can be$ expressed as a finite range integral:

$$
\begin{aligned}
\phi_{x}(\omega)= & \prod_{k=1}^{L}\left[\frac{\exp \left(-K_{k}\right)}{\pi} \int_{0}^{\pi} \exp \left(\left(\frac{j \omega \xi+\sqrt{2 K_{k}} \cos \theta}{2}\right)^{2}\right)\right. \\
& \left.\times D_{-2}\left(-j \omega \xi-\sqrt{2 K_{k}} \cos \theta\right) d \theta\right]
\end{aligned}
$$

In particular, the development of (4) circumvents the need to compute the confluent hypergeometric functions recursively (i.e., Eqs. (12) - (14) in [6]). Now using variable substitution $t=\cos \theta$ and then applying the GCQ formula [12, (25.4.38)], we have yet another series expression for the CHF of $x$ in a Rician fading channel,

$$
\begin{aligned}
\phi_{x}(\omega) \cong \prod_{k=1}^{L}[ & \frac{\exp \left(-K_{k}\right)}{n} \sum_{i=1}^{n} \exp \left(\left(\frac{j \omega \xi+\sqrt{2 K_{k}} \cos x_{i}}{2}\right)^{2}\right) \\
& \times D_{-2}\left(-j \omega \xi-\sqrt{2 K_{k}} \cos x_{i}\right)
\end{aligned}
$$

where $x_{i}=(2 i-1) \pi / 2 / n$. Note that there is a trade-off involved in the choice of the value of $n$. A greater accuracy may be obtained using a larger value of $n$, but at the expense of increased number of terms. A bound on the approximation error for the truncated series is also provided in [11].

\section{Rayleigh Fading}

Substituting $m_{k}=1$ in (2) or $K_{k}=0$ in (4) for all $k \in\{1, \ldots, L\}$, we get the CHF of $x$ in a Rayleigh fading, i.e.,

$$
\phi_{x}(\omega)=\prod_{k=1}^{L}\left[\Phi\left(1, \frac{1}{2} ; \frac{\bar{\gamma}_{k} \omega^{2}}{4 L}\right)+j \omega \sqrt{\frac{\pi \bar{\gamma}_{k}}{4 L}} \exp \left(\frac{-\bar{\gamma}_{k} \omega^{2}}{4 L}\right)\right]
$$

\section{ERROR PROBABILITY ANALYSIS}

Table 1 summarizes the instantaneous SER for a wide range of modulation schemes in an AWGN channel. Recognizing the alternative exponential form for the complementary error functions, i.e., $\quad \operatorname{erfc}(\sqrt{\gamma})=\frac{2}{\pi} \int_{0}^{\pi / 2} \exp \left(-\gamma \csc ^{2} \theta\right) d \theta \quad$ and $\operatorname{erfc}^{2}(\sqrt{\gamma})=\frac{4}{\pi} \int_{0}^{\pi / 4} \exp \left(-\gamma \csc ^{2} \theta\right) d \theta$, we can express these conditional error probabilities (for binary and M-ary signal constellations) as a special case of the following generic form,

$$
P_{S}(\varepsilon \mid \gamma)=\sum_{k} \int_{0}^{\eta_{k}} a_{k}(\theta) \exp \left(-\gamma b_{k}(\theta)\right) d \theta
$$

where $a_{k}(\theta)$ and $b_{k}(\theta)$ are coefficients independent of $\gamma$ but may be dependent on $\theta$. Since $\gamma=x^{2}$, we can rewrite (7) as

$$
P_{S}(\varepsilon \mid x)=\sum_{u} \int_{0}^{\eta_{u}} a_{u}(\theta) \exp \left(-x^{2} b_{u}(\theta)\right) d \theta
$$


The average bit or symbol error probability (ASER) in fading channels can be obtained by averaging the conditional error probability over the PDF of the combined signal amplitude at the output of the EGC combiner, namely

$$
P_{s}=\int_{0}^{\infty} P_{s}(\varepsilon \mid x) p_{x}(x) d x
$$

where $p_{x}($.$) denotes the PDF of RV x$.

\section{A. CHF Method using Parseval's Theorem}

If the fading amplitudes are assumed to be independent, then the evaluation of ASER using the classical solution in the form of (9) will require an $L$-fold convolution integral. It is more insightful if we transform the PDF into frequency domain since the CHF of $x$ (i.e., sum of $L$ fading amplitudes) is simply the product of the individual CHFs. However, it is difficult (or impossible) to invert $\phi_{x}($.$) to get a closed-form$ expression for the PDF of $x$. Therefore, a Fourier series approach has previously been used $[5,6]$.

Using the inverse Fourier transform representation for the PDF, and then rearranging the order of integration, (9) can be restated as

$$
\begin{aligned}
P_{S} & =\int_{0}^{\infty} P_{S}(\varepsilon \mid x)\left[\frac{1}{2 \pi} \int_{-\infty}^{\infty} \phi_{x}(\omega) \exp (-j \omega x) d \omega\right] d x \\
& =\frac{1}{2 \pi} \int_{-\infty}^{\infty} \phi_{x}(\omega)\left[\int_{0}^{\infty} P_{S}(\varepsilon \mid x) \exp (-j \omega x) d x\right] d \omega \\
& =\frac{1}{2 \pi} \int_{-\infty}^{\infty} \mathrm{FT}\left[P_{S}(\varepsilon \mid x)\right] \phi_{x}^{*}(\omega) d \omega
\end{aligned}
$$

where notation $\phi_{x}{ }^{*}($.$) denotes the complex conjugate of the$ CHF of $x$.

In fact, the final result of $(10)$ follows directly from the application of Parseval's theorem [14, pp. 371] to transform the product integral in (9) into the frequency domain, thereby circumventing the need to find the PDF of $x$. But we then also need the FT of $P_{S}(\varepsilon \mid \bar{x})$, which surprisingly turns out to be very easily computed.

The FT of the generic conditional error probability (i.e., Eq. (8)) is given by

$$
\begin{aligned}
\dot{G}(\omega)= & \sum_{u} \int_{0}^{n_{u}} a_{u}(\theta) \int_{0}^{x} \exp \left\{-x^{2} b_{u}(\theta)+j \omega x\right\} d x d \theta \\
= & \frac{1}{2} \sum_{u} \int_{0}^{n_{u}} \frac{a_{u}(\theta)}{b_{u}(\theta)}\left[\sqrt{\pi b_{u}(\theta)} \exp \left(\frac{-\omega^{2}}{4 b_{u}(\theta)}\right)\right. \\
& \left.+j \omega \Phi\left(1, \frac{3}{2} ; \frac{-\omega^{2}}{4 b_{u}(\theta)}\right)\right] d \theta
\end{aligned}
$$

Substituting (11) into (10), and realizing that the imaginary part of this integral is zero (since the ASER is real), we get an exact analytical SER expression for binary and M-ary modulation formats with predetection EGC:

$$
P_{S}=\frac{1}{\pi} \int_{0}^{\pi} \operatorname{Real}\left\{G(\omega) \phi_{x}^{*}(\omega)\right\} d \omega=\frac{2}{\pi} \int_{0}^{\pi / 2} \frac{\psi(\tan \zeta)}{\sin (2 \zeta)} d \zeta
$$

where $\psi(\omega)=\operatorname{Real}\left\{\omega G(\omega) \phi_{x}{ }^{*}(\omega)\right\}$. Notice that the evaluation of (12) for the most general case involves two-fold inte-

\begin{tabular}{|c|c|}
\hline Modulation Scheme & Conditional Error Probability $P_{S}(\varepsilon \mid \gamma)$ \\
\hline \multicolumn{2}{|l|}{ Coherent binary signalling: } \\
\hline (a) Coherent PSK & $0.5 \operatorname{erfc}(\sqrt{\gamma})$ \\
\hline $\begin{array}{l}\text { (b) Coherent detection of } \\
\text { differentially encoded PSK }\end{array}$ & $\operatorname{erfc}(\sqrt{\gamma})-\frac{1}{2} \operatorname{erfc}^{2}(\sqrt{\gamma})$ \\
\hline (c) Coherent FSK & $0.5 \operatorname{erfc}(\sqrt{\gamma / 2})$ \\
\hline \multicolumn{2}{|l|}{ Noncoherent binary signalling: } \\
\hline (a) DPSK & $0.5 \exp (-\gamma)$ \\
\hline (b) Noncoherent FSK & $0.5 \exp (-\gamma / 2)$ \\
\hline \multicolumn{2}{|l|}{ Quadrature signalling: } \\
\hline (a) QPSK & $\operatorname{erfc}(\sqrt{\gamma})-0.25 \operatorname{erfc}^{2}(\sqrt{\gamma})$ \\
\hline (b) MSK & $\operatorname{erfc}(\sqrt{\gamma})-0.25 \operatorname{erfc}^{2}(\sqrt{\gamma})$ \\
\hline $\begin{array}{l}\text { (c) } \pi / 4-D Q P S K \text { with Gray } \\
\text { coding }\end{array}$ & $\begin{array}{l}\frac{1}{2 \pi} \int_{0}^{\pi} \frac{\exp (-\gamma(2-\sqrt{2} \cos \theta))}{\sqrt{2}-\cos \theta} d \theta \\
\text { or } \frac{1}{2 \pi} \int_{0}^{\pi} \exp \left(\frac{-2 \gamma}{2-\sqrt{2} \cos \theta}\right) d \theta\end{array}$ \\
\hline \multicolumn{2}{|l|}{ Multilevel signalling: } \\
\hline (a) Square QAM & $\begin{array}{l}2 q \operatorname{erfc}(\sqrt{p \gamma})-q^{2} \operatorname{erfc}^{2}(\sqrt{p \gamma}) \\
q=1-1 / \sqrt{M}, p=1.5 \log _{2} M /(M-1)\end{array}$ \\
\hline (b) MPSK & $\frac{1}{\pi} \int_{0}^{\pi-\pi / M} \exp \left(\frac{-\gamma \sin ^{2}(\pi / M) \log _{2} M}{\sin ^{2} \theta}\right) d \theta$ \\
\hline (c) MDPSK [16] & $\frac{1}{\pi} \int_{0}^{\pi-\pi / M} \exp \left(\frac{-\gamma \sin ^{2}(\pi / M) \log _{2} M}{1+\cos (\pi / M) \cos \theta}\right) d \theta$ \\
\hline $\begin{array}{l}\text { (d) Two-dimensional signal } \\
\text { constellations }[17,10]\end{array}$ & $\begin{array}{l}\frac{1}{2 \pi} \sum_{u=1}^{N} \operatorname{Pr}\left(S_{u}\right) \int_{0}^{n_{u}} \exp \left(\frac{-\gamma \alpha \sin ^{2}\left(\Psi_{u}\right)}{\sin ^{2}\left(\theta+\Psi_{u}\right)}\right) d \theta \\
\text { where } N \text { is the number of signal points, and } \\
\operatorname{Pr}\left(S_{u}\right) \text { is the a priori probability that the } u \text { th } \\
\text { signal point is transmitted. }\end{array}$ \\
\hline
\end{tabular}
grals. Next -we will identify four special cases of the conditional error probability $P_{s}(\varepsilon \mid x)$ which allow the evalu- ation of the generic expression given in (12) to be further simplified into a single finite-range integral. This simplification is attributed to the availability of closed-form formulas for the FT of $P_{S}(\varepsilon \mid x)$.

Table 1. Instantaneous SER of several common modulation schemes.

\section{A.l Exponential Form: $P_{S}(\varepsilon \mid x)=a \exp \left(-b x^{2}\right)$}

The instantaneous BER of some noncoherent binary modulation schemes (e.g., DPSK and NCFSK) can be expressed in the exponential form. In this case, the FT of $a \exp \left(-b x^{2}\right)$ is given by,

$$
G(\omega)=\frac{a}{\sqrt{b}}\left[\frac{\sqrt{\pi}}{2} \exp \left(\frac{-\omega^{2}}{4 b}\right)+j F\left(\frac{\omega}{2 \sqrt{b}}\right)\right]
$$

where $F($.$) denotes the Dawson integral,$

$$
F(x)=\exp \left(-x^{2}\right) \int_{0}^{x} \exp \left(t^{2}\right) d t=x \Phi\left(1, \frac{3}{2} ;-x^{2}\right)
$$

Dawson's integral can be computed more efficiently using a direct method (based on sampling theorem) suggested by Rybicki [15] instead of evaluating sufficiently large number of terms in the series representation of $\Phi\left(1, \frac{3}{2} ;-x^{2}\right)$. For this reason, we have expressed $G(\omega)$ in terms of the Dawson's integral. The corresponding ASER is obtained by substituting (13) into (12). 
Now using variable substitution $\omega^{2}+\frac{1}{2}=\frac{1}{1+x}$ in (12) and then applying GCQ formula [12], we get a rapidly converging series representation for the EGC receiver performance on generalized fading channels,

$$
P_{S}=\frac{1}{n} \sum_{k=1}^{n} \Psi\left[\frac{1}{\sqrt{2}} \tan \left(\frac{\pi(2 k-1)}{4 n}\right)\right] / \sin \left(\frac{\pi(2 k-1)}{2 n}\right)+R_{n}
$$

where $R_{n}$ denotes the remainder term. A simple bound for $R_{n}$ is also derived in [11] using a complex-variable method.

\section{A.2 Complementary Error Function: $P_{S}(\varepsilon \mid x)=a \operatorname{erfc}(\sqrt{b} x)$}

The conditional error probability of some coherent binary modulation schemes (e.g., CPSK and CFSK) are in the form of complementary error function. The FT of $a \operatorname{erfc}(\sqrt{b} x)$ can be shown as

$$
G(\omega)=\frac{a}{\omega}\left[\frac{2}{\sqrt{\pi}} F\left(\frac{\omega}{2 \sqrt{b}}\right)+j\left(1-\exp \left(\frac{-\omega^{2}}{4 b}\right)\right)\right]
$$

Therefore, the ASER can be computed efficiently by substituting (16) into (12) or (15).

$$
\text { A.3 } P_{s}(\varepsilon \mid x)=\operatorname{aerfc}(\sqrt{b} x)-c \operatorname{erfc}^{2}(\sqrt{b} x)
$$

The instantaneous SER for QPSK, MQAM and coherent detection of differentially encoded PSK can be expressed in the form $a \operatorname{erfc}(\sqrt{b} x)-c \operatorname{erfc}^{2}(\sqrt{b} x)$. Utilizing the results of (16) and recognizing that the FT of $c \operatorname{erfc}^{2}(\sqrt{b} x)$ term can derived using integration by parts, we get

$$
\begin{aligned}
& G(\omega)=\frac{2 a}{\omega \sqrt{\pi}} F\left(\frac{\omega}{2 \sqrt{b}}\right)-\frac{4 c}{\omega \sqrt{\pi}}\left[F\left(\frac{\omega}{2 \sqrt{b}}\right)-F\left(\frac{\omega}{2 \sqrt{2 b}}\right) \exp \left(\frac{-\omega^{2}}{8 b}\right)\right] \\
& +j\left[\frac{a}{\omega}\left(1-\exp \left(\frac{-\omega^{2}}{4 b}\right)\right)-\frac{c}{\omega}\left(1-\exp \left(\frac{-\omega^{2}}{4 b}\right)-\frac{4}{\pi} F^{2}\left(\frac{\omega}{2 \sqrt{2 b}}\right)\right)\right](17) \\
& A .4 P_{S}(\varepsilon \mid x)=\Gamma\left(a, b x^{2}\right) / 2 \Gamma(a)
\end{aligned}
$$

In [18], Wojnar presents a unified bit error rate expression (due to Linder) for coherent, differentially coherent and noncoherent detection of binary signals transmitted over AWGN channel (see Table 2),

$$
P_{h}(\varepsilon \mid x)=\frac{\Gamma\left(a, b x^{2}\right)}{2 \Gamma(a)}
$$

\begin{tabular}{|c|c|c|}
\hline$b \quad a$ & $1 / 2$ & 1 \\
\hline $1 / 2$ & Orthogonal CFSK & Orthogonal NCFSK \\
\hline 1 & Antipodal CPSK & Antipodal DPSK \\
\hline
\end{tabular}

where $\Gamma(.,$.$) denotes the complementary incomplete Gamma$ function, and $\Gamma($.$) is the Gamma function.$

Table 2. Parameters $a$ and $b$ for different binary modulation formats and detection schemes.

Using identity [13, (6.452.1)] and after some algebraic manipulations, the FT of $P_{b}(\varepsilon \mid x)$ can be shown as

$$
G(\omega)=\frac{j}{\omega}\left[\frac{1}{2}-\frac{\Gamma(2 a)}{\Gamma(a) 2^{4}} \exp \left(\frac{-\omega^{2}}{8 b}\right) D_{-2 a}\left(\frac{-j \omega}{\sqrt{2 b}}\right)\right]
$$

or alternatively,

$$
\begin{aligned}
G(\omega)= & \frac{\Gamma(2 a)}{[\Gamma(a)]^{2} 2^{2 a}} \sqrt{\frac{\pi}{b}} \Phi\left(\frac{1}{2}+a, \frac{3}{2} ; \frac{-\omega^{2}}{4 b}\right) \\
& +\frac{j}{\omega}\left[\frac{1}{2}-\frac{\Gamma(2 a) \sqrt{\pi}}{\Gamma(a) \Gamma(a+1 / 2) 2^{2 a}} \Phi\left(a, \frac{1}{2} ; \frac{-\omega^{2}}{4 b}\right)\right]
\end{aligned}
$$

For the special cases of $a=1$ and $a=1 / 2,(20)$ reduces to the familiar form similar to (13) and (16), respectively.

\section{B. PDF Method using Fourier Series Approximation}

Although it is difficult to invert $\phi_{x}($.$) to get the PDF of x$ in a closed-form, we can easily derive an approximate of the desired PDF using a Fourier series technique. Let us assume $0 \leq x<T$ (i.e., upper limit of $x$ is limited to $T$ although it has an infinite range) where $T$ is selected such that $\operatorname{Pr}(x>T) \leq \varepsilon$, and $\varepsilon$ can be set to a very small value. Therefore, the PDF of $x$ can be expressed by a Fourier series,

$$
p_{x}(x)=\sum_{n=-\infty}^{\infty} c_{n} e^{j n \omega_{0} x}
$$

where $\omega_{0}=2 \pi / T$ and

$$
c_{n}=\frac{1}{T} \int_{0}^{T} p_{x}(x) e^{-j n \omega_{0} x} d x \approx \frac{1}{T} \phi_{x}\left(-n \omega_{0}\right)
$$

by our assumption. Thus substituting (21) into (9), we have

$$
P_{S} \cong \frac{1}{T} \sum_{n=-\infty}^{\infty} G\left(n \omega_{0}\right) \phi_{x}\left(-n \omega_{0}\right)
$$

where $G($.$) is the FT of P_{S}(\varepsilon \mid x)$ as before. Notice that the final result (23) is equivalent to [5, Eq. (29b)] or [10, Eq. (14)] but in a slightly different form.

\section{CHF Method with a Desirable Exponential Integral Form for the Gaussian Probability Integral}

Since $Q(x)$ is the tail probability of a zero-mean, unit variance Gaussian random variable (GRV) exceeds $x$, and the CHF of GRV is $\phi(t)=\exp \left(-t^{2} / 2\right)$, we immediately get

$$
Q(x)=\frac{1}{2}-\frac{1}{\pi} \int_{0}^{x} \frac{1}{t} e^{-t^{2} / 2} \sin (t x) d t
$$

by invoking the Gil-Pelaez inversion theorem (Fourier inversion formula). This new form is suitable for performing the average over the distribution of the fading amplitudes. Hence, an exact analytical expression for the coherent binary signalling schemes with EGC is given by

$$
\begin{aligned}
P_{b} & =\frac{1}{2}-\frac{1}{\pi} \int_{0}^{\infty} t^{-1} e^{-t^{2} / 2} \operatorname{Imag}\left\{\phi_{x}(2 \sqrt{a} t)\right\} d t \\
& =\frac{1}{2}-\frac{1}{2 \pi} \int_{0}^{x} t^{-1} e^{-t} \operatorname{Imag}\left\{\phi_{x}(2 \sqrt{a} t)\right\} d t
\end{aligned}
$$

where $a=1$ for CPSK and $a=1 / 2$ for CFSK.

\section{PDF Method using Hermite Integral Approximation}

In [8], the authors presented yet another approximate solution for BPSK with EGC receiver. Using the PDF method with Hermite integral approximation, the ASER of the generic error probability (8) (i.e., for binary and M-ary modulation formats) with predetection EGC may be computed as, 


$$
\begin{aligned}
P_{S} \cong \frac{1}{2 \pi} \sum_{u} \int_{0}^{\eta_{u}} a_{u}(\theta) \sum_{n=1}^{H} w_{n} \text { Real }\left\{\phi_{x}\left(2 x_{n} \sqrt{b_{u}(\theta)}\right)\right. \\
\left.\times\left[\sqrt{\pi}-2 j x_{n} \Phi\left(\frac{1}{2}, \frac{3}{2} ; x_{n}^{2}\right)\right]\right\} d \theta
\end{aligned}
$$

where $x_{n}$ and $w_{n}$ are the $n$-th abscissa and weight respectively of the $H$-th order Hermite polynomial [12].

Eq. (26) generalizes the result found in [8] for an arbitrary two dimensional signal constellations. It is further pointed out that the computational complexity of (26) for BPSK, QPSK and MQAM modulation formats is actually much poorer than the CHF methods or the Fourier series approach because (26) involves an evaluation of a double integral.

\section{E. Closed-Form Solutions for EGC in Nakagami Fading}

In [7], Zhang formulated the problem of computing the ABER for coherent binary PSK with EGC diversity in the framework of statistical decision theory, and then obtained closed-form solutions for $L \leq 3$ in a Rayleigh fading channel. By contrast, here we derive new closed-form formulas for the ABER with EGC in Nakagami fading via the CHF methods (IIIA and IIIC). While the results are restricted to positive integer values of the fading severity index, $m_{k}$ need not be identical. If we further assume that $m_{k}$ is common to all the diversity branches, then we can also get closed-form formula for the case $m=0.5$ with two or order-three diversity. A detailed treatment of this problem is discussed in [11].

For binary CPSK and CFSK; the performance of nondiversity system (i.e., $L=1$ ) in a Nakagami fading channel with arbitrary $m$ can be readily shown as

$$
P_{S}=\frac{1}{2}-\sqrt{\frac{\bar{\gamma}}{(m \delta+\bar{\gamma}) \pi}} \frac{\Gamma \cdot(m+1 / 2)}{\Gamma(m)}{ }_{2} F_{1}\left(1-m, \frac{1}{2} ; \frac{3}{2} ; \frac{\bar{\gamma}}{m \delta+\bar{\gamma}}\right)
$$

where ${ }_{2} F_{1}(., \ldots ;)$ denotes the Gauss hypergeometric function, $\delta=1$ for BPSK, and $\delta=2$ for BFSK. For $L=2$, we can show that the ABER for binary CPSK and CFSK is given by

$$
P_{S}=\frac{1}{2}-I_{2}(1,2, \delta)-I_{2}(2,1, \delta)
$$

where

$$
\begin{aligned}
& I_{2}(x, y, \delta)=\frac{\Gamma\left(m_{x}+1 / 2\right)}{\pi \Gamma\left(m_{x}\right)} \sum_{k=0}^{m_{y}-1}\left(\begin{array}{c}
\left.m_{x}-1\right) \\
k
\end{array}\right) \frac{\Gamma(k+1 / 2)}{(2 k+1) ! !} \\
& \quad \times(-2)^{k}\left[\frac{\gamma_{x} m_{y}}{\bar{\gamma}_{x} m_{y}+\bar{\gamma}_{y} m_{x}+2 m_{x} m_{y} \delta}\right]^{k+\frac{1}{2}} \\
& \quad \times{ }_{2} F_{1}\left(\frac{1}{2}-m_{y}, k+\frac{1}{2} ; \frac{1}{2} ; \frac{\bar{\gamma}_{y} m_{x}}{\bar{\gamma}_{x} m_{y}+\bar{\gamma}_{y} m_{x}+2 m_{x} m_{y} \delta}\right)
\end{aligned}
$$

Substituting $m_{x}=m_{r}=1$ in (28) and recognizing that ${ }_{2} F_{1}(a, b ; b ; x)=(1-x)^{-a}$, we get

$$
P_{S}=\frac{1}{2}-\frac{1}{2}\left[\frac{\sqrt{\bar{\gamma}_{1}\left(\bar{\gamma}_{1}+2 \delta\right)}+\sqrt{\bar{\gamma}_{2}\left(\bar{\gamma}_{2}+2 \delta\right)}}{\bar{\gamma}_{1}+\bar{\gamma}_{2}+2 \delta}\right]
$$

which is identical to Eq. (23) in [7]. Using this idea, one may also derive a closed-form expression for EGC in Nakagami fading with three diversity branches. Closed-form expression for noncoherent binary modulation formats in Nakagami fading is derived in [11] for $L=2$ case.

\section{CONCLUSIONS}

This paper presents a concise, unified approach to evaluate the performance of predetection equal-gain diversity for a broad class of modulation formats and fading environments. Our results are sufficiently general to allow for arbitrary fading parameters as well as dissimilar mean signal strengths across the diversity branches. The generality and computational efficiency of the new results presented in this paper rendering themselves as powerful means for both theoretical analysis and practical applications. Some new closed-form solutions for the EGC receiver performance in Nakagami fading environment are also derived.

\section{REFERENCES}

[1] W. C. Jakes, Microwave Mobile Communications, NY: Wiley, 1974.

[2] N. C. Beaulieu, "An Infinite Series for the Computation of the Complementary Probability Distribution Function of a Sum of Independent Random Variables and Its Application to the Sum of Rayleigh Random Variables," IEEE Trans. Commun., Vol. 38, pp. 1463-1474, Sept. 1990.

[3] F. J. Altman and W. Sichak, "A Simplified Diversity Communication System for Beyond the Horizon Links," IRE Trans. Commun. Systems, Vol. 4, pp. 50-55, March 1956.

[4] M. Schwartz, W. R. Bennett and S. Stein, Communication Systems and Techniques, New York: McGraw-Hill, 1966.

[5] N.C. Beaulieu and A. Abu-Dayya, "Analysis of Equal Gain Diversity on Nakagami Fading Channels," IEEE Trans. Commun., Vol. 39, pp. 225-234, February 1991.

[6] A. Abu-Dayya and N. C. Beaulieu, "Microdiversity on Rician Fading Channels," IEEE Trans. Commun., Vol. 42, June 1994, pp. 2258-2267.

[7] Q. T. Zhang, "Probability of Error for Equal-gain Combiners over Rayleigh Channels: Some Closed-Form Solutions," IEEE Trans. Commun., Vol. 45, March 1997, pp. 270-273.

[8] M. Simon and M. Alouini, "A Unified Approach to Performance Analysis of Digital Communication over Generalized Fading Channels," Proc. IEEE, Vol. 86, Sept. 1998, pp. 1860-1877.

[9] A. Annamalai, C. Tellambura and V. K. Bhargava, "Exact Evaluation of Maximal-Ratio and Equal-Gain Diversity Systems for MQAM on Nakagami Fading Channels," to appear in the IEEE Trans. Commun.

[10] X. Dong, N. C. Beaulieu and P. H. Wittke, "Two Dimensional Signal Constellations for Fading Channels," Proc. IEEE Globecom'98 Communication Theory Mini Conference, pp. 22-27.

[11] A. Annamalai, C. Tellambura and V. K. Bhargava, "Unified Analysis of Equal-Gain Diversity Systems on Rician and Nakagami Fading Channels," manuscript in preparation for journal submission.

[12] M. Abramowitz and I. A. Stegun, Handbook of Mathematical Functions, National Bureau of Standards, 1964.

[13] I. S. Gradshteyn and I. M. Ryzhik, Table of Integrals, Series and Products, Academic Press, 5th edition, 1995.

[14] P. Z. Peebles, Probability, Random Variables and Random Signal Principles, McGraw-Hill, 1993.

[15] G. B. Rybicki, "Dawson's Integral and Sampling Theorem," Computers in Physics, Vol. 3; pp. 85-87, March 1989.

[16] R. F. Pawula, "A New Formula for MDPSK Symbol Error Probability" IEEE Communications Letters, Vol. 2, pp. 271-272, Oct. 1998.

[17] J. W. Craig, "A New, Simple and Exact Results for Calculating the Probability of Error for Two Dimensional Signal Constellations," IEEE MILCOM'9l Conference Record, pp. 25.5.1-25.5.5.

[18] A. H. Wojnar, "Unknown Bounds on Performance in Nakagami Channels," IEEE Trans. Communications, Vol. 34, Jan. 1986, pp. 22-24. 